\title{
The Effects of Interdistracter Similarity on Search Processes in Superior Parietal Cortex
}

\author{
David T. Wilkinson,* Peter W. Halligan,† Richard N. A. Henson,‡ and Raymond J . Dolanł’ \\ *Department of Experimental Psychology, Oxford University, Oxford OX1 3UD, United Kingdom; †School of Psychology, \\ Cardiff University, Cardiff CF 10 3YG, United Kingdom; ¥Wellcome Department of Cognitive Neurology \& Institute \\ of Cognitive Neuroscience, University College London, London WC1N 3BG, United Kingdom; and \\ §Royal Fre Hospital School of Medicine, Roland Hill Stree, London NW3 2DF, United Kingdom
}

Received March 14, 2001

The superior parietal lobe has been associated with the spatial integration of visual features, an important step in the detection of particular form conjunctions. However, behavioral research has indicated that when target items can be segmented from neighboring distracters via similarity grouping, detection may not rely on spatial integration. The question therefore arises as to whether the superior parietal cortex is an integral component of conjunction search or only important in the absence of certain grouping relations. Here, we acquired measures of reaction time and event-related fMRI, while subjects searched for conjunction targets in displays containing either homogeneous or heterogeneous distracters. We confirm that under conditions of low distracter similarity, search involves parietal-motor areas associated with spatial selection. However, we also demonstrate that under conditions of high distracter similarity, search is instead associated with activation of right temporal-parietal cortex. These results suggest that the superior parietal cortex is not a necessary component of visual conjunction search and highlight a new role for the right temporal-parietal cortex in perceptual grouping. ๑ 2002 Elsevier Science (USA)

\section{INTRODUCTION}

The superior parietal cortex (SPC) has been strongly implicated in visuospatial selection. Relative to appropriate baselines, strong activation in the superior parietal lobule and neighboring intraparietal sulcus is typically observed in healthy observers when they must shift their attention to peripheral locations (i.e., Corbetta, 1988; Corbetta and Shulman, 1988; Corbetta et al., 1993, 2000), irrespective of whether this is accompanied by either an eye movement or a manual response (Corbetta et al., 1997), whether the cue to shift is either endogenous or exogenous (Kim et al., 1999; N obre et al., 1997), or whether the task requires target detection (Coull and Nobre, 1998) or identification (Kim et al., 1999; Nobre et al., 1997). Patients with damage extending to this region often neglect the presence of stimuli appearing in their contralesional field (i.e., De Renzi, 1982; Mesulam, 1981) and experience difficulty in disengaging and subsequently redirecting attention from one location to another (Egly et al., 1994; Posner et al., 1984).

One particular task in which the SPC has been shown to play a role is visual conjunction search, in which subjects must report the presence or absence of a target that is defined by a unique combination of features. SPC involvement is consistent with the proposal that the spatial locations of features must be coded before these can be bound into integrated forms (although it remains unclear if this spatial coding is performed in either a serial or a parallel fashion) (Cave and Wolfe, 1990; Reynolds and Desimone, 1999; Treisman and Gelade, 1980; Wolfe, 1994; Yantis and J ohnson, 1990). Studies in healthy observers have shown that search for conjunctions defined by either color and motion or color and orientation, relative to single-feature search, preferentially engages SPC, while singlefeature search, relative to conjunction search, engages processes primarily located in the occipital and temporal cortices (Corbetta et al., 1993, 1995; Donner et al., 2000; Hunton et al., 1995). Patients with damage to this region are prone to perceiving illusory conjunctions, in which features from different locations are erroneously combined, and are abnormally slow in identifying conjunctions but not features (Cohen and Rafal, 1991; Friedman-Hill et al., 1995; Humphreys et al., 1985; Robertson et al., 1997). Likewise, broad disruption to this area, via transcranial magnetic stimulation, has been shown to interfere with conjunction, but not feature, search (Ashbridge et al., 1997). These findings could be taken to suggest that SPC is a necessary component of conjunction search.

However, one aspect of the conjunction searches described above is that displays always contain distract- 
ers that are heterogeneous. This is important because behavioral evidence shows that the degree of distracter similarity can moderate search performance; when distracters are heterogeneous the time taken to identify a target increases with greater numbers of display items, whereas when distracters share the same identity, search rates are relatively unaffected by display size (Duncan and Humphreys, 1989, 1992; Humphreys et al., 1989; Müller et al., 1994; von Grünau et al., 1994). These different display size effects have been taken to reflect the operation of underlying search mechanisms that are differentially sensitive to the spatial location of stimuli. In particular, it has been proposed that distracters which share the same identity can be grouped via preattentive mechanisms that are sensitive to the gestalt property of similarity. Although models differ in the precise manner in which this kind of grouping might support efficient search (see, for example, models by Duncan and Humphreys, 1992; Reynolds and Desimone, 1999; Treisman and Gelade, 1980; Wolfe, 1994), there is a consensus that under these grouping conditions, the target is detected as an "odd man out." In this form of search, the presence/ absence of a target can be deduced by the presence/ absence of a single, disjunctive item that disrupts the global homogeneity of displays. By contrast, where grouping relations are either absent or do not segment the target from distracters, items must be individually checked for their target status. Many have suggested that this form of inspection requires the spatial locations of stimuli to be attended (i.e., Friedman-Hill and Wolfe, 1995; Treisman, 1998; Treisman and Gelade, 1980; Treisman and Sato, 1990; Wolfe, 1994). Critically, if it can be shown that nonspatial conjunction search (i.e., where distracters are homogeneous) activates regions other than SPC, then evidence will have been found that the SPC is not a necessary component of conjunction search per se, but rather a product of particular interdistracter similarity relations.

The brain areas supporting search for conjunctions surrounded by homogeneous distracters are poorly understood, although previous research has indicated that the cerebral hemispheres do not code distracter similarity relations equally (Donnelly and Wilkinson, 1999). I n this study, subjects searched for an upright $T$ set among homogeneous distracters, maximizing both the grouping between nontargets and the differences between targets and distracters. Displays were briefly presented unilaterally and led to a consistent pattern of errors; error rates increased linearly with display size in the right visual field-left hemisphere (RVF-LH), but not in the left visual field-right hemisphere (LVF$\mathrm{RH})$. These findings are consistent with the idea that search is affected by similarity relations operating only in the right hemisphere. However, it remains unclear if these behavioral effects reflect, at the level of neural activation, either a right hemisphere bias or the oper- ation of areas other than superior parietal cortex. One clue comes from the observation that while superior parietal/intraparietal lesions are typically associated with spatial deficits (Holmes, 1918; Posner et al., 1984), impairments in the analysis and identification of visual form often relate to damage to inferior parietal and temporal cortices (Farah, 1990; Lamb et al., 1989, 1990; Newcombe and Russell, 1969).

To investigate the modulatory effects of distracter similarity on superior parietal cortex in visual search, subjects in the present study searched for a conjunction target (upright $\mathrm{T}$ ) set among distracters that were either homogeneous (all upside down T's) or heterogeneous (randomly oriented T's). There was also a baseline condition involving search for a single feature (/) among distracters $(\backslash)$ to enable examination of those areas conjointly activated by both forms of conjunction search. Event-related fMRI data were acquired from subjects while the search tasks were performed. In line with previous findings, we predicted that in the heterogeneous condition, subjects would show display size effects and accordingly, relative to the homogeneous condition, search should activate parietal-motor structures associated with shifts in spatial attention (Nobre et al., 1997). However, in the homogeneous condition target detection would instead be reliant on similarity grouping and display size effects smaller. We predicted that this would show a right hemisphere bias, most likely focused within inferior parietal/temporal regions.

\section{METHOD}

\section{Subjects}

Seven male and five female, normal-sighted subjects, with a mean age of 29, were tested. All were right handed, yielding a group handedness mean of 21 of a maximum of 24, as tested by the Briggs and Nebes (1975) modified version of the Annett's Handedness Inventory.

\section{Stimuli}

Stimuli appeared black on a white background (see Fig. 1) at a viewing distance of approximately $35 \mathrm{~cm}$. The T stimuli subtended $1.2^{\circ}$ in both height and width, with a thickness of $0.3^{\circ}$. The / stimuli measured $1.3^{\circ}$ with a thickness of $0.3^{\circ}$. In the T conditions, the target was an upright $\mathrm{T}$, and distracters were either all upside down (homogeneous condition) or oriented at angles $90^{\circ}, 180^{\circ}$, and $270^{\circ}$ from vertical (heterogeneous condition). Targets in the single-feature condition were oriented at $45^{\circ}$, while distracters were oriented at $315^{\circ}$. Target present trials consisted of only a single target appearing, with the number of distracters varying be tween 1 and 4 across displays. Display items were organized in a vertical column, and the distance be 
A

B C

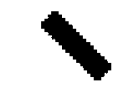

$\perp$

$\therefore$

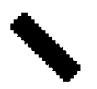
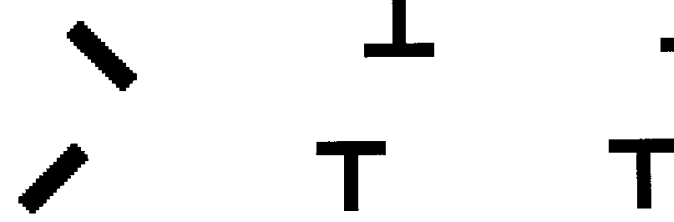

FIG. 1. Example stimuli from (A) single-feature displays and (B and $\mathrm{C}$ ) conjunction displays. Distracters in the conjunction displays were either homogeneous (B) or heterogeneous (C). Displays contained 2, 3, 4 (depicted here), or 5 items. Targets are lowermost in each example, but were randomly distributed across item location in the actual experiment.

tween each item was $1^{\circ}$. To lend an element of unpredictability into displays, for half of all trials, every second item from the top was shifted $0.8^{\circ}$ to the left, while for the remainder items were shifted $0.8^{\circ}$ to the right. Likewise, the midpoint of displays was displaced approximately $5^{\circ}$ left or right of the center of the screen. Screen center was marked with a central fixation cross measuring $1.5 \times 1.5^{\circ}$.

\section{Procedure}

Subjects were shown pictures of the targets and informed that these would appear in different blocks and that their task was to report, as quickly but as accurately as possible, whether for each stimulus display a target was present or absent. They were also told to maintain central fixation following the appearance of the fixation cross and throughout stimulus presentation.

Subjects were then given three blocks of practice trials, showing an equal number of trials from each stimulus by display size by target present/absent condition. Subjects signaled their response either by pressing the "J " and "G" computer keys in the practice session conducted outside the scanner or by pressing one of two response buttons on a pad held with one hand during scanning. Responding hand and the mapping between response button and present/absent response were counterbalanced across subjects.

The three stimulus conditions were run in 10 separate, pseudo-randomly ordered blocks, with display size and present/absent trials randomly manipulated within each block. Within each block of trials, the target appeared eight times, and each display size condition appeared four times. Each block of 16 trials began with an instruction screen, showing a picture of the target, with the word "Target" placed directly above. This remained on the screen for $3 \mathrm{~s}$. Targets appeared randomly (but the same number of times overall) at each possible item location. Trials began with a fixation cross for 500 ms, followed by the stimulus for 200 ms and a stimulus onset asynchrony of $2.5 \mathrm{~s}$ during which time the screen remained blank. Each display size condition contained 40 trials (20 present/20 absent), generating a total of 480 trials for the whole experiment.

\section{Image Acquisition}

A 2-T Magnetom Vision system was used to acquire both 3D MP-RAGE sequence T1 anatomical images $(1 \times 1 \times 1.5-\mathrm{mm}$ voxels) and T2*-weighted EPI images (3 $\mathrm{mm}$ in-plane resolution with a slice thickness of 2 $\mathrm{mm}$ and a gap of $1 \mathrm{~mm}$, yiel ding a resolution of $3 \times 3 \times$ $3 \mathrm{~mm}$ ). Each EPI image comprised 32 1.8-mm axial slices and employed a TE of $40 \mathrm{~s}$. Four hundred ninety volume images were acquired with a TR of $3.2 \mathrm{~s} / \mathrm{vol}$ ume. Each subject's scans were realigned to the first volume and resliced using sinc interpolation, adjusting for residual motion-related signal changes. Data were also interpolated temporally to the middle slice acquired. A mean image was then coregistered with a structural T1 volume and then spatially normalized to a standard template. The data were smoothed using a 10-mm (full width at half-maximum) isotropic Gaussian kernel.

\section{fMRI Data Analysis}

Data were analyzed using statistical parametric mapping (SPM99) employing a two-stage procedure, implementing a mixed-effects analysis. In the first level design matrix, we specified 12 potential effects of interest, consisting of each Stimulus (single feature, $T$ with homogeneous distracters, $T$ with heterogeneous distracters) by Display Size $(2,3,4,5)$ combination. These effects were modeled by convolving a delta function at each stimulus onset with a canonical hemodynamic response function to create regressors of interest. The mixed-effects analysis involved two stages. First, session-specific parameter estimates pertaining to the hemodynamic response function for each effect of interest were calculated for each voxel and images of contrasts of these parameter estimates calculated for each subject. These contrast images were then entered into a second analysis (one sample t test), treating subjects as a random variable. Statistical effects in the heterogeneous and homogeneous contrast are reported at $\mathrm{P}<0.001$, uncorrected for multiple comparisons, when associated with the predicted bilateral parietalfrontal network or right inferior parietal/temporal lobe regions, respectively. All other effects were thresholded at $\mathrm{P}<0.05$, corrected. 


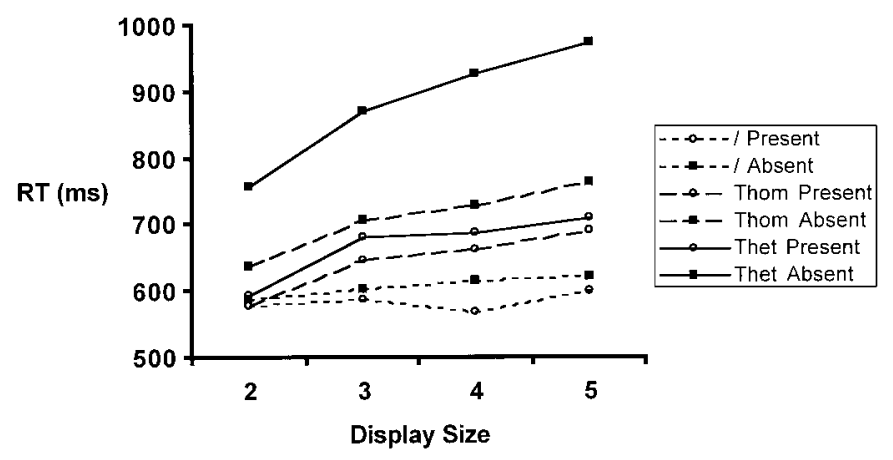

FIG. 2. Mean correct reaction times (ms) as a function of display size and target present/absent for single-feature targets (/) and conjunction targets presented among either homogeneous (Thom) or heterogeneous (Thet) distracters.

\section{RESULTS}

\section{Behavioral Data}

Mean correct reaction time (RT) and arcsine transformed errors were computed for each subject. RTs that were more than 2.5 standard deviations from the mean were discarded. RT and accuracy scores were analyzed in a separate 3 (Distracter Type: single feature vs homogeneous conjunction vs heterogeneous conjunction) $\times 2$ (Target: present vs absent) $\times 4$ (Display Size: 2 vs 3 vs 4 vs 5) ANOVA repeated over all factors. Post hoc analyses were conducted using planned comparisons.

There were significant main effects of Distracter Type $(\mathrm{F}(2,22)=79.4, \mathrm{MSe}=19,700, \mathrm{P}<0.01)$, Target $(\mathrm{F}(1,11)=64.8, \mathrm{MSe}=22,593, \mathrm{P}<0.01)$, and Display Size $(F(3,33)=41.2, \mathrm{MSe}=6647, \mathrm{P}<0.01)$. There were also reliable interaction effects between Distracter Type and Target $(\mathrm{F}(2,22)=30.6, \mathrm{MSe}=15,736$, $P<0.01)$, Distracter Type and Display Size $(F(6,66)=$ 13.5, $\mathrm{MSe}=3470, \mathrm{P}<0.01)$, Target and Display Size $(\mathrm{F}(3,33)=3.3, \mathrm{MSe}=5063, \mathrm{P}<0.05)$, and Distracter Type, Target, and Display Size $(\mathrm{F}(6,66)=2.3$, MSe $=$ 2875, $\mathrm{P}<0.05$ ) (see Fig. 2).

The error data reproduced the main effects of Distracter Type $(\mathrm{F}(2,22)=18.5, \mathrm{MSe}=2.0, \mathrm{P}<0.01)$ and Display Size $(\mathrm{F}(3,33)=42.4, \mathrm{MSe}=0.5, \mathrm{P}<0.01)$, as well as the interactions between Distracter Type and Display Size $(F(6,66)=13.8, \mathrm{MSe}=0.5, \mathrm{P}<0.01)$ and Distracter Type, Display Size, and Target $(F(6,66)=$ 3.5, $\mathrm{MSe}=0.5, \mathrm{P}<0.01$ ).

Overall, responses were slowest in the heterogeneous conjunction condition and fastest (and flattest) in the single-feature condition. In accordance with previous studies, displays containing heterogeneous distracters generated absent responses (71 ms per item) that increased approximately linearly and at a ratio of 2:1 to present trials ( $35 \mathrm{~ms}$ per item). By contrast, slope ratios for absent (40 ms per item) and present (35 ms per item) responses in conjunction displays containing homogeneous distracters were approximately equal and much shallower. Present/absent responses in the heterogeneous condition were respectively slower than present/absent responses in the homogeneous condition. The two forms of response were broadly equated in the single-feature condition (present, $12 \mathrm{~ms}$ per item; absent, 5 ms per item).

These data suggest that search rates were strongly affected by both target and distracter identity. The relatively shallow slopes derived from conjunction displays containing homogeneous distracters are in line with previous studies and highlight the potential role of preattentive grouping in conjunction search (Humphreys et al., 1989; Treisman, 1998; Wolfe, 1994). This mode of search differs from "pop-out" search, in which, although display homogeneity can also be used to detect targets, responses can be based solely on the presence of a single, unique feature (i.e., Treisman and Gelade, 1980; Wolfe, 1994). The small linear component to responses in the homogeneous condition may, in part, be accounted for by the restricted viewing conditions; stimuli were presented unilaterally for only $200 \mathrm{~ms}$. Previous research has indicated that search may be less efficient when there is a rapid decay of visual information (Klein and Farrell, 1989) and when displays are presented slightly into the periphery (Duncan and Humphreys, 1989).

One issue raised by a reviewer concerned the reduced difference in target present, relative to absent, responses across the two forms of conjunction search. In particular, it was suggested that both forms of search might have been mediated by the same underlying mechanism. In fact, present responses were significantly slower in the heterogeneous, relative to the homogeneous, condition, which poses a problem for any such unitary account. One reason for the relatively fast present responses in the heterogeneous condition may have related to the identity of the target. Unlike distracter items, the target was a highly familiar, alphanumeric character and did not vary in its physical form. This may have led to a very fast target comparison rate, relative to the distracter comparison rate, and narrowed the difference between present responses across the two display conditions (see Townsend and Ashby, 1983). Faster present trials for heterogeneous displays may also have been influenced by a process of "clumping," which has been shown to operate at small display sizes (Pashler, 1987). Here, individual elements are conjointly selected as part of a larger subset of display items and inspected in parallel, after which search then moves, in a serial manner, to the next predefined clump. If subjects did search in this way, then the linearly increasing slopes associated with absent trials may have reflected an additional item-by-item error checking process, encouraged by the low target-distracter discriminability (Chun and Wolfe, 1996). Although clumping can reduce display 
TABLE 1

Contrasts Associated with Significant Brain Activation showing Region (Brodmann's Area), Side, Talairach Coordinates of Maximum Activation ( $\mathrm{mm})$ and Z Score

\begin{tabular}{|c|c|c|c|c|c|c|}
\hline \multirow[b]{2}{*}{ Contrast } & \multirow[b]{2}{*}{ Activation (BA) } & \multirow[b]{2}{*}{ Side } & \multicolumn{3}{|c|}{ Coordinates } & \multirow[b]{2}{*}{ Z score } \\
\hline & & & & $(x, y, z)$ & & \\
\hline \multirow[t]{2}{*}{ Thet-baseline } & Temporal-parietal junction (39) & $\mathrm{R}$ & 46 & -70 & 28 & 3.9 \\
\hline & & $\mathrm{R}$ & 46 & -64 & 22 & 3.8 \\
\hline \multirow[t]{10}{*}{ Thet-Thom } & M1 (4) & $\mathrm{R}$ & 22 & -16 & 56 & 4.3 \\
\hline & & L & -26 & -24 & 46 & 4.0 \\
\hline & Cerebellum & $\mathrm{R}$ & 36 & -38 & -26 & 3.7 \\
\hline & & $\mathrm{L}$ & -10 & -76 & -18 & 4.0 \\
\hline & Superior parietal lobe (7) & $\mathrm{R}$ & 24 & -70 & 42 & 3.5 \\
\hline & & $\mathrm{L}$ & -8 & -80 & 36 & 3.6 \\
\hline & SMA (6) & $R / L$ & 4 & 2 & 54 & 3.8 \\
\hline & Pulvinar & $\mathrm{R}$ & 20 & -20 & 8 & 3.7 \\
\hline & Superior occipital gyrus (19) & $\mathrm{R}$ & 26 & -80 & 28 & 3.9 \\
\hline & Inferior occipital gyrus (18) & $\mathrm{R}$ & 32 & -90 & -4 & 3.7 \\
\hline Thet 5-Thet 2 & SMA (6) & $\mathrm{R}$ & 18 & 8 & 70 & 3.7 \\
\hline Thom-baseline & Anterior insula (11) & $\mathrm{R}$ & 36 & 28 & -6 & 4.5 \\
\hline \multirow[t]{8}{*}{ Thet-baseline } & M1 (4) & L & -38 & -16 & 46 & 4.0 \\
\hline & Premotor (6) & $\mathrm{R}$ & 20 & -16 & 74 & 4.7 \\
\hline & Cerebellum & $\mathrm{R}$ & 42 & -80 & 24 & 4.0 \\
\hline & & L & -10 & -74 & -12 & 4.8 \\
\hline & Superior parietal lobe (7) & $\mathrm{R}$ & 24 & -78 & 24 & 4.3 \\
\hline & & L & -24 & -70 & 36 & 4.4 \\
\hline & Inferior occipital gyrus (19) & $\mathrm{R}$ & 40 & -84 & 8 & 4.1 \\
\hline & Anterior insula (11) & $\mathrm{R}$ & 30 & 28 & -12 & 5.2 \\
\hline
\end{tabular}

Note. Results are thresholded at an extent of 30 voxels and height of $\mathrm{P}<0.05$ corrected for multiple comparisons, unless specified otherwise in the text.

size effects, the important issue here is that it does not do so by utilizing the global display properties afforded by gestalt grouping. I n shifting between clumps, it also employs a spatially selective element. In this way, present responses across the two forms of conjunction search may still have derived from separate mechanisms, even though they bore similar search functions.

In fact, it is notoriously difficult to associate either RT or error slopes with a particular mode of search, since different processes can generate similar search slopes (Townsend and Ashby, 1983). In the present context, we merely wish to emphasize that the behavioral data broadly replicated the reported effects of interdistracter similarity on search efficiency. Of course, the principal issue is whether these distinct patterns of search dissociate at the neural level.

\section{Imaging Data}

Simultaneously acquired event-related fMRI data provided strong evidence for the modulatory role of distracter similarity in visual search. Condition-specific increases in brain activity are shown in Table 1. Searching through conjunction displays containing homogeneous distracters relative to heterogeneous distracters activated regions around right temporal-parietal junction (rTPJ) (see Fig. 3). This activation was inferior to the intraparietal sulcus (IPS) and extended between superior temporal and angular gyri. No activation was observed elsewhere (all $\mathrm{P}$ values exceeded 0.001 , uncorrected for multiple comparisons). Relative to the baseline single-feature condition, the only significant area of activation for homogeneous distracter search was in the right anterior insula.

By contrast, search for an upright $\mathrm{T}$ among heterogeneous distracters, relative to homogeneous distracters, activated regions normally associated with spatial shifts of attention (Nobre et al., 1997); bilateral superior parietal lobe (including IPS), bilateral motor cortex, supplementary motor area, and left cerebellum (see Fig. 4). Further evidence of sequential processing in the heterogeneous condition was observed by an effect of display size, whereby right supplementary motor area was activated to greater effect for display sizes of 5 relative to 2 . Relative to the baseline condition, search in heterogeneous distracter displays again produced bilateral parietal-motor activation; activity was observed in bilateral cerebellum, right premotor cortex, left motor cortex, bilateral superior parietal cortex, right inferior occipital gyrus, and right anterior insula.

\section{DISCUSSION}

The data indicate that when distracters can be segmented from the target via similarity grouping, detec- 

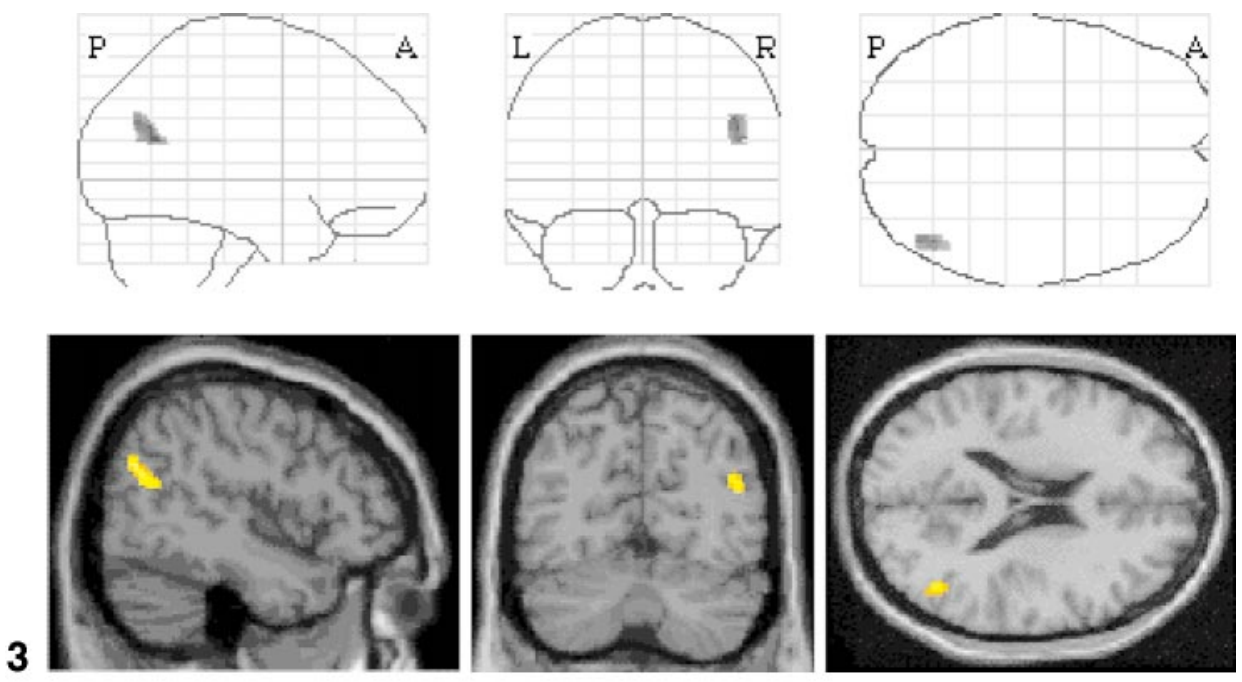

sagittal

coronal

transverse
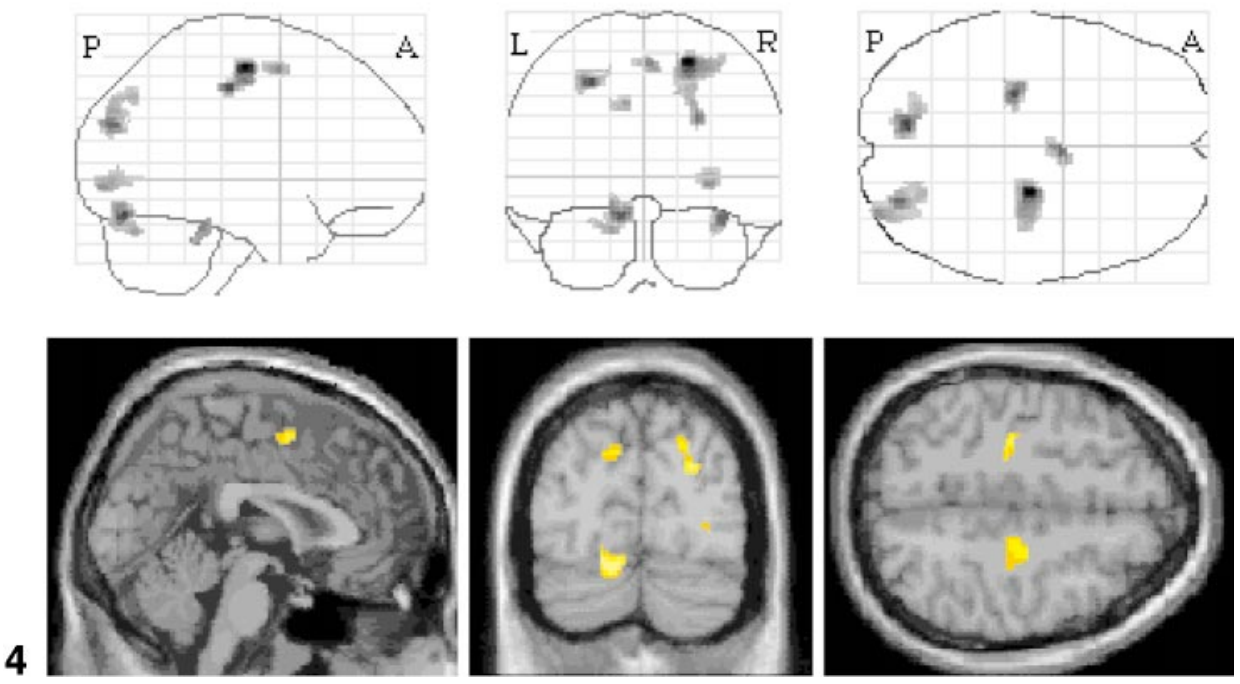

FIG. 3. Activation of right temporal-parietal junction during search for an upright $T$ in a field of inverted T's, relative to search in a field of randomly oriented T's. The top depicts activation as through projections onto representations of standard stereotactic space (A, anterior; $P$, posterior; L, left; $R$, right). The bottom depicts this activation rendered onto a canonical MRI structural image.

FIG. 4. Activation of the bilateral parietal-motor network associated with search for an upright $T$ in a field of randomly oriented T's, relative to search in a field of inverted T's.

tion of conjunction targets involves rTPJ. In the absence of these grouping relations, search is mediated by superior parietal-motor regions associated with spatial selection (Corbetta et al., 1995). These effects of distracter similarity are reflective of the role of perceptual grouping in visual conjunction search and constitute new evidence that it is not the mere search for conjunction targets that activates the superior, posterior parietal lobe. Rather, it is the failure of grouping mechanisms to preattentively segment target from distracter items and the subsequent need for feature binding that engages superior parietal cortex. We emphasize that these findings may not hold in other kinds of displays in which high interdistracter similarity is ac- companied by high target-distracter similarity. It also remains to be seen if TPJ is activated in other kinds of conjunction search, in which although grouping does not segment target from distracters, distracters are highly similar or search functions relatively flat (see Wolfe, 1994).

The inclusion of the baseline condition enabled those regions conjointly activated by both kinds of conjunction search, relative to single feature search, to be examined. The data revealed conjoint activation of right anterior insula. This region is strongly implicated in affective processing (Rolls, 1999), and in the absence of further experimentation, the activation may be best viewed as indexing the heightened emotion associated 
with greater task difficulty in the conjunction searches. There was significant parietal-motor activation in both heterogeneous- homogeneous and heterogeneous- baseline contrasts. Since the baseline condition may be taken as a measure of nonspatial search, this overlap further supports claims that targets in the homogeneous condition were detected using a nonspatial mechanism. This is also supported by the failure to obtain distinct patterns of activation in visual prestriate cortex for the homogeneous vs baseline contrast.

The involvement of rTPJ in target detection is consistent with data from other visual tasks. Using a spatial cueing paradigm, Corbetta et al . (2000) showed that while IPS was strongly implicated in the voluntary orienting of attention to a cued location, detection of targets located at an uncued attention preferentially activated rTPJ. This selectivity to target, rather than cue, information may also be nonspatial in nature; strong activation was observed in an "oddball" task, in which subjects had to report the occurrence of a new object (at the same location) in strings of rapidly occurring stimuli (it should be noted that strong activation was also observed when a familiar stimulus appeared at a novel location) (Marois et al., 2000). This led the authors to suggest that this region may be involved in the perceptual, automatic stimulus-driven aspects of visual selection, rather than in top-down attentional control. The current data are in agreement with this proposed role in nonspatial selection and extend it by showing a preattentive sensitivity to similarity relations.

Coupled with findings from these other studies, it is tempting to believe that there are separable functional visual areas within rTPJ . Performance on the different tasks described above mapped onto discrete anatomical foci; both sets of rTPJ Talairach coordinates in the Corbetta et al. (2000) study $(57,-45,12)(53,-49,30)$, lay lateral and anterior to the coordinates reported in the current study $(46,-70,20)$, as did the coordinates $(53,-34,21)$ in the Marois et al . (2000) study. There is also some neuropsychological support for a dissociation of function: visual neglect, in which the patient is unable to orient or act upon contralesional stimuli, often arises following rTPJ damage (Vallar and Perani, 1986). However, other rTPJ patients may show little or no neglect, yet show impairment in the identification of global form in compound stimuli (Robertson et al., 1988). Other lesions can induce visual dysgnosia, in which distortions to egocentric space disrupt the viewer's perceived location, relative to other environmental cues (Cogan, 1979, but see also Bottini, 2001). Also, tracer studies in monkeys reveal subdivisions in TPJ which contain distinct patterns of afferent and efferent connections to other visual areas. Within the region there is extensive local, segregated processing; the caudal third of inferior parietal lobule projects to three architectonic areas in superior temporal sulcus (STS), the midinferior parietal lobe projects to the caudal upper bank of STS only, while rostral inferior parietal lobe does not project to temporal lobe (Seltzer and Pandya, 1984). More distally, area 7a reciprocally projects to STS, while 7ip (in IPS) connects to different superior temporal regions, including the ventral bank, V5, and fundal superior temporal regions (Neal et al., 1990). Regions located in extrastriate visual and prefrontal cortices also project to different sites in STS, including the polysensory area TPO and those regions adjacent to V5 (Seltzer et al., 1996). These findings are perhaps unsurprising given both the generic nature of the term TPJ and the fact that the region has no tightly defined anatomical boundary. Nonetheless, the existence of several functional areas within rTPJ may help explain its involvement in a variety of seemingly disparate visual tasks and associated disorders.

Lateralization of similarity grouping to the right hemisphere is consistent with the visual field effect reported by Donnelly and Wilkinson (1999), in which search rates were nonlinear in LVF-RH but linearly increasing in RVF-LH. The identification of cerebral asymmetries at this early level is important since many forms of visual operation may be based on these initial grouped representations (Marr, 1982; Treisman, 1998). In this sense, asymmetries observed in more complex, attentional tasks may in part be reducible to hemispheric differences of this kind. For example, the right hemisphere has long been associated with the processing of gestalt property (Bradshaw and Nettleton, 1981; Van Kleeck and Kosslyn, 1991), particularly in the identification of gl obal form in compound stimuli (Lamb et al., 1989). Recent study has shown that gestalt factors (including similarity) play a role in the construction of global percepts in Navon stimuli (Han et al., 1999) while others have shown that patients with damage to the rTPJ are abnormally slow in identifying targets defined at the global, relative to the local, level. These patients also fail to show the normal interference effects from global forms when identifying local forms (Rafal and Robertson, 1995; Robertson et al., 1988). In demonstrating that rTPJ is sensitive to grouping via similarity, the current results therefore provide a tentative link between the computation of global form from gestalt factors and global processing deficits following rTPJ damage.

Previous studies have shown significant overlap in the neural mechanisms that mediate saccadic eye movement and shifts in covert spatial attention (i.e., Corbetta, 1998; Kim et al., 1999; Nobre et al., 1997). One question that arises is whether the parietal-motor activation in the heterogeneous condition reflected greater eye movement. Although eye movements were recorded inside the scanner, difficulties with the software prevented their analysis. Nonetheless, there are several reasons to believe that eye movements did not play a major role in the parietal-motor activation. 
First, subjects were instructed to maintain gaze at a central fixation point. Trial-by-trial observation of eye gaze confirmed that subjects were properly fixated for the majority of trials. Second, in order to minimize eye movement, stimulus exposure duration was restricted to $200 \mathrm{~ms}$-too little time for subjects to saccade to lateral stimuli. Third, eye movements have been shown to consistently activate the frontal eye fields, which are located laterally in premotor area 6 between the central sulcus and the precentral sulcus (see Nobre et al., 1997). In the current study, all activations were posterior to the central sulcus, with the exception of a single midline foci in the supplementary motor area, which was evident in the heterogeneous- homogeneous contrast. It should be noted that the primary aim of the current study was to examine, within a visual search task, if changes to the perceptual arrangement of displays generated distinct patterns of activation. I rrespective of whether the spatial shifts of attention, as indexed by parieto-motor activation, were covert or overt, it remains the case that search through grouped, relative to ungrouped, distracters, modulated superior parietal activity.

In sum, we confirm previous claims that the superior parietal lobe plays an important role in conjunction search. However, we show for the first time that it is not the mere search for conjunction targets that activates the superior parietal lobe but the combined failure of similarity grouping and subsequent need to independently access the identity of each item. In addition, we demonstrate that when distracter similarity is high, search is mediated by a region in rTPJ . These findings highlight a new role for rTPJ in the coding of gestalt property.

\section{ACKNOWLEDGMENTS}

D.T.W. and P.W.H. are supported by the Medical Research Council. R.N.A.H. and R.J .D. are supported by the Wellcome Trust.

\section{REFERENCES}

Ashbridge, E., Walsh, V., and Cowey, A. 1997. Temporal aspects of visual search studied by transcranial magnetic stimulation. Neuropsychologia 35: 1121-1131.

Bottini, G., Karnath, H., Vallar, G., Sterzi, R., Frith, C., Frackowiak, R. S. J ., and Paulesu, E. 2001. Cerebral representations of egocentric space: Functional-anatomical evidence from caloric stimulation and neck vibration. Brain 124: 1182-1196.

Bradshaw, J. L., and Nettleton, N. C. 1981. The nature of hemispheric specialization in man. Behav. Brain Sci. 4: 51-91.

Briggs, G. G., and Nebes, R. D. 1975. Patterns of hand preference in a student population. Cortex 11: 230-238.

Cave, K. R., and Wolfe, J. M. 1990. Modeling the role of parallel processing in visual search. Cognit. Psychol. 22: 225-271.

Chun, M. M., and Wolfe, J. M. 1996. J ust say No: How are visual searches terminated when there is no target present? Cognit. Psychol. 30: 39-78.
Cohen, A., and Rafal, R. 1991. Attention and feature integration: Illusory conjunctions in a patient with a parietal lobe lesion. Psychol. Sci. 2: 206-110.

Cogan, D. G. 1979. Visual spatial dysgnosia. Am. J . Ophthalmol . 88: 361-388.

Corbetta, M. 1998. Frontoparietal networks for directing attention and the eye to visual locations: I dentical, independent, or overlapping neural systems? Proc. Natl. Acad. Sci. USA 95: 831- 838.

Corbetta, M., and Shulman, G. L. 1998. Human cortical mechanisms of visual attention during orienting and search. Philos. Trans. R. Soc. London B Biol. Sci. 353: 153-1362.

Corbetta, M., Shulman, G. L., Miezin, F. M., and Petersen, S. E. 1993. A PET study of visuospatial attention. J. Neurosci. 13: 1202-1226.

Corbetta, M., Shulman, G. L., Miezin, F. M., and Petersen, S. E. 1995. Superior parietal cortex activation during spatial attention shifts and visual feature conjunction. Science 270: 802- 805.

Corbetta, M., Shulman, G. L, Conturo, T. E., Snyder, A. Z., Akbudak, E., Petersen, S. E., and Raichle, M. E. 1997. Functional magnetic resonance imaging (fMRI) of visuospatial attention: Group and single subject analysis. Neurol mage 5(Suppl.): 85.

Corbetta, M., Kincade, J. M., Ollinger, J. M., McAvoy, M. P., and Shulman, G. L. 2000. Voluntary orienting is dissociated from target detection in human posterior parietal cortex. Nat. Neurosci. 3: 292-297.

Coull, J . T., and Nobre, A. C. 1998. Where and when to pay attention: The neural systems for directing attention to spatial locations and to time intervals as revealed by both PET and FMRI. J . Neurosci. 18: $7426-7435$.

De Renzi, E. 1982. Disorders of Space Exploration and Cognition. Wiley, New York.

Donnelly, N., and Wilkinson, D. 1999. Searching for targets in the left and right visual fields. Brain Cognit. 40: 104-108.

Donner, T., Kettermann, A., Diesch, E., Ostendorf, F., Villringer, A., and Brandt, S. A. 2000. I nvolvement of the human frontal eye field and multiple parietal areas in covert visual selection during conjunction search. Eur. J. Neurosci. 12: 3407-3414.

Duncan, J ., and Humphreys, G. W. 1989. Visual search and stimulus symmetry. Psychol. Rev. 96: 453- 458.

Egly, R., Driver, J ., and Rafal, R. D. 1994. Shifting visual attention between objects and locations: Evidence from normal and parietal lesion subjects. J . Exp. Psychol. Gen. 123: 161-177.

Farah, M. J . 1990. Visual Agnosia: Disorders of Object Recognition and What They Tell Us about Normal Vision. MIT Press/Bradford Books, Cambridge, MA.

Friedman-Hill, S. R., Robertson, L. C., and Treisman, A. 1995. Parietal contributions to visual feature binding: Evidence from a patient with bilateral lesions. Science 269: 853- 855.

Han, S., Humphreys, G. W., and Chen, L. 1999. Uniform connectedness and classical gestalt principles of perceptual grouping. Percept. Psychophys. 61: 661- 674.

Holmes, G. 1918. Disturbances of visual orientation. Br. J . Ophthalmol. 2: 449-468.

Humphreys, G. W., Riddoch, M. J ., and Quinlan, P. T. 1985. Interactive processes in perceptual organization: Evidence from visual agnosia. In Attention and Performance (M. I. Posner and S. M. Marin, Eds.), Vol. XI, pp. 301-318. Erlbaum, Hillsdale, NJ .

Humphreys, G. W., Quinlan, P. T., and Riddoch, M. J . 1989. Grouping processes in visual search: Effects with single- and combinedfeature targets. J . Exp. Psychol. Gen. 118: 258-279.

Hunton, D. L., Corbetta, M., Shulman, G. L., Miezin, F. M., and Petersen, S. E. 1995. Common areas of parietal activations for shifts of spatial attention and tasks involving the conjunction of visual features. Soc. Neurosci. Abstr. 21: 937. 
Kim, Y. H., Gitelman, D. R., Nobre, A. C., Parrish, T. B., LaBar, K. S., and Mesulam, M. M. 1999. The large-scale neural network for spatial attention displays multifunctional overlap but differential asymmetry. Neurol mage 9: 269-277.

Klein, R., and Farrell, M. 1989. Search performance without eye movements. Percept. Psychophys. 46: 476- 482.

Lamb, M. R., Robertson, L. C., and Knight, R. T. 1989. Attention and interference in the processing of hierarchical patterns: Inferences from patients with right and left temporal-parietal lesions. Neuropsychologia 17: 619-627.

Lamb, M. R., Robertson, L. C., and Knight, R. T. 1990. Component mechanisms underlying the processing of hierarchically organised patterns: I nferences from patients with unilateral cortical lesions. J . Exp. Psychol. LMC 16: 471- 483.

Marois, R., Leung, H. C., and Gore, J. C. 2000. A stimulus-driven approach to object identity and location processing in the human brain. Neuron 25: 717-728.

Marr, D. 1982. Vision. Freeman, San Francisco.

Mesulam, M. 1990. Large-scale neurocognitive networks and distributed processing for attention, language, and memory. Ann. Neurol . 28: 597- 613.

Müller, H. J ., Humphreys, G. W., and Donnelly, N. 1994. Search via recursive rejection (SERR): Visual search for single and dual form conjunction targets. J . Exp. Psychol. HPP 20: 235-238.

Neal, J . W., Pearson, R. C., and Powell, T. P. 1988. The cortico-cortico connections within the parieto-temporal lobe of area PG, 7a, in the monkey. Brain Res. 438: 343-350.

Newcombe, F., and Russell, W. 1969. Dissociated visual perceptual and spatial deficits in focal lesions of the right hemisphere. J . Neurol. Neurosurg. Psychiatry 32: 73-81.

Nobre, A. C., Sebestyen, G. N., Gitelman, D. R., Mesulam, M. M, Frackiowiak, R. S., and Frith, C. D. 1997. Functional localization of the system for visuo-spatial attention using positron emission tomography. Brain 120: 515-333.

Pashler, H. 1987. Detecting conjunctions of colour and form: Reassessing the serial search hypothesis. Percept. Psychophys. 41: 191201.

Posner, M. I., Walker, J. A., Freidrich, F. J., and Rafal, R. 1984. Effects of parietal lobe injury on covert orienting of visual attention. J . Neurosci. 4: 1863-1874.

Rafal, R., and Robertson, L. C. 1995. The neurology of visual attention. In Handbook of Cognitive Neuroscience (M. Gazzaniga, Ed.). MIT Press, Cambridge, MA.

Reynolds, J. H., and Desimone, R. 1999. The role of neural mechanisms of attention in solving the binding problem. Neuron 24: 19-29.
Robertson, L. C., Lamb, M. C., and Knight, R. T. 1988. Effects of lesions of temporal-parietal junction on perceptual and attentional processing in humans. J . Neurosci. 8: 3757-3769.

Robertson, L. C., Treisman, A., Friedman-Hill, S. R., and Grabowecky, M. 1997. The interaction of spatial and object pathways: Evidence from Balint's syndrome. J . Cognit. Neurosci. 9: 295-317.

Rolls, E. T. 1999. The Brain and Emotion. Oxford Univ. Press, Oxford.

Seltzer, B., and Pandya, D. N. 1984. Further observations on parietotemporal connections in the rhesus monkey. Exp. Brain Res. 55: 301-312.

Seltzer, B., Cola, M. G., Gutierrez, C., Massee, M., Weldon, C., and Cusick. C. G. 1996. Overlapping and nonoverlapping cortical projections to cortex of the superior temporal sulcus in the rhesus monkey: Double anterograde tracer studies. J . Comp. Neurol . 370: 173-190.

Townsend, J. T., and Ashby, G. F. 1983. The Stochastic Modeling of Elementary Psychol ogical Processes. Cambridge Univ. Press, Cambridge, UK.

Treisman, A. 1988. Feature and objects: The Fourteenth Bartlett Memorial Lecture. Q. J . Exp. Psychol. A40: 201-237.

Treisman, A. 1998. Feature binding, attention and object perception. Philos. Trans. R. Soc. London B Biol. Sci. 353: 1295-306.

Treisman, A. 1999. Solutions to the binding problem: Progress through controversy and convergence. Neuron 24: 105-110.

Treisman, A., and Gelade, G. 1980. A feature-integration theory of attention. Cognit. Psychol. 12: 97-136.

Treisman, A., and Sato, S. 1990. Conjunction search revisited. J . Exp. Psychol. HPP 16: 459-478.

Vallar, G., and Perani, D. 1986. The anatomy of unilateral neglect after right hemisphere stroke lesions. A clinical/CT-scan correlation study in man. Neuropsychologia 24: 609- 622.

Van Kleeck, M. H., and Kosslyn, S. M. 1989. Gestalt laws of perceptual organization in an embedded figures task: Evidence for hemispheric specialization. Neuropsychologia 27: 1165-1178.

Von Grünau, M., Dubé, S., and Galera, C. 1994. Local and global factors of similarity in visual search. Percept. Psychophys. 55: 575-592.

Wolfe, J. M. 1994. Guided search 2.0. A revised model of visual search. Psychonom. Bull. Rev. 1: 202-238.

Wolfe, J. M., and Cave, K. R. 1990. Modeling the role of parallel processing in visual search. Cognit. Psychol. 22: 225-271.

Yantis, S., and J ohnson, D. N. 1990. Mechanisms of attentional priority. J . Exp. Psychol. HPP 16: 812-825. 